\title{
Holographic complexity of charged Taub-NUT-AdS black holes
}

\author{
Jie Jiang,,$^{2, *}$ Banglin Deng, ${ }^{1, \dagger}$ and Xiao-Wei Li $\oplus^{3, *}$ \\ ${ }^{1}$ Department of Applied Physics, College of Geophysics, Chengdu University of Technology, \\ Chengdu 610059 Sichuan, China \\ ${ }^{2}$ Department of Physics, Beijing Normal University, Beijing 100875, China \\ ${ }^{3}$ School of Physics, University of Chinese Academy of Sciences, Beijing 100049, China
}

(Received 6 August 2019; published 6 September 2019)

\begin{abstract}
In this paper, we investigate the holographic complexity in the charged Taub-NUT-AdS black holes with Misner strings present in the Einstein-Maxwell gravity. We show that differing from the normal black holes, where the late-time complexity growth rate is determined only by the quantities at outer and inner "Reissner-Nordstrom"-type (RN-type) horizons, here the quantities (the Misner potential and Misner charge) related to the Misner strings also play an important role in "complexity equals action" (CA) complexity. Similar to the case of the normal electromagnetic black hole, the late-time rate for the original CA conjecture is independent on the magnetic charges. However, disparate with common results of the dyonic solutions, the electric charge that appeared here is the total charge of this black hole. Besides, we found that the result in this original CA conjecture also violates the electromagnetic duality. And this duality can be restored by adding the Maxwell boundary term with the proportional constant $\gamma=1 / 2$. In this case, the late-time rate is sensitive to the magnetic charge. Moreover, we also found that the additional term changes only the proportion between the electric and magnetic charges, and it does not affect the Misner term that appeared in the late-time rate. Finally, we studied the time dependence of the complexity growth rate and found that they share similar behaviors with that in RN-AdS black holes.
\end{abstract}

DOI: 10.1103/PhysRevD.100.066007

\section{INTRODUCTION}

In recent years, there has been a growing interest in the topic of "quantum complexity," which is defined as the minimum number of gates required to obtain a target state starting from a reference state [1,2]. From the holographic viewpoint, Brown et al. suggested that the quantum complexity of the state in the boundary theory is dual to some bulk gravitational quantities which are called "holographic complexity." Then, the two conjectures, "complexity equals volume" (CV) $[2,3]$ and "complexity equals action" (CA) [4,5], were proposed. They aroused widespread attention of researchers to both holographic complexity and circuit complexity in quantum field theory, e.g., [6-66].

In the present work, we focus on only the CA conjecture, which states that the quantum complexity of a particular state $\left|\psi\left(t_{L}, t_{R}\right)\right\rangle$ on the boundary is given by

\footnotetext{
*jiejiang@mail.bnu.edu.cn

Corresponding author.

bldeng@aliyun.com

"lixiaowei16@mails.ucas.ac.cn

Published by the American Physical Society under the terms of the Creative Commons Attribution 4.0 International license. Further distribution of this work must maintain attribution to the author(s) and the published article's title, journal citation, and DOI. Funded by SCOAP .
}

$$
C_{A}\left(\left|\psi\left(t_{L}, t_{R}\right)\right\rangle\right) \equiv \frac{I_{\mathrm{WDW}}}{\pi \hbar} .
$$

Here $I_{\text {WDW }}$ is the on-shell action in the corresponding Wheeler-DeWitt (WDW) patch, which is enclosed by the past and future light sheets sent into the bulk spacetime from the time slices $t_{L}$ and $t_{R}$. As argued in Ref. [4], there is a bound of the complexity growth rate at the late time

$$
\dot{C} \leq \frac{2 M}{\pi \hbar},
$$

which may be thought as the Lloyd's bound of the black hole system [67].

In the previous works, the CA conjecture in the normal black holes for a variety of gravitational theories has been investigated. In Ref. [6], based on the Noether charge formalism introduced by Iyer and Wald, a general formalism has been obtained to describe the late-time CA complexity growth rate in a multiple-horizon black hole for a general F (Riemann) gravity, i.e.,

$$
\lim _{t \rightarrow \infty} \frac{d C_{A}}{d t}=\frac{1}{\pi \hbar}\left[\Omega^{(\mu)} J_{(\mu)}+\Lambda_{\infty}[\xi]-\Lambda_{\mathcal{C}}[\xi]\right]_{+}^{-},
$$

where $\Omega^{(\mu)}$ and $J_{(\mu)}$ are the angular velocity and angular momentum associated with the Killing horizon, respectively, 
$\Lambda_{\mathcal{C}}$ denotes some charges related to the matter field, and the index $\{ \pm\}$ presents the quantities evaluated at the "outer" or "inner" horizon. This result implies that the late-time rate depends on only the quantities on the horizons. And this can be understood since the boundary of the WDW patch is bounded only by the spheres $\Sigma_{ \pm}$on the outer or inner horizon and $S_{\infty}$ at asymptotic infinity. However, there are also some black holes with different topology than a normal black hole, such as the Taub-NUT-AdS black hole with the Misner strings $[68,69]$. How is this nontrivial topology reflected in the holographic complexity? For this reason, the purpose of this paper is to study the effect of the nontrivial topology of the spacetime on the holographic complexity in the CA picture.

As one of the most interesting solutions of general relativity, Taub-NUT spacetime $[68,69]$ has engendered lots of investigations since birth. In this spacetime, apart from the Reissner-Nordstrom (RN)-type horizons, there also exist two Misner string singularities on the north and south pole axes due to the existence of Newman-UntiTamburino (NUT) parameter $n$. This Misner string is also a Killing horizon of this black hole, and it will actually change the topology of the spacetime geometry, especially the late-time boundaries of the WDW patch. As illustrated by Refs. [70,71], there exist two quantities to describe the Misner string: the Misner potential $\psi$ and charge $N$, which is introduced to make the thermodynamics of the TaubNUT black hole have the expected features. In the rest of this paper, we would like to evaluate the CA complexity growth rate and discuss its corrections caused by this Misner string.

Our paper is organized as follows. In the next section, we briefly review the rudiments about the charged Taub-NUTAdS spacetime and enumerate some basic thermodynamic quantities for this black hole. In Sec. III, we evaluate the time dependence of the complexity growth rate in the charged Taub-NUT-AdS black hole in the original CA picture and discuss the affection of the Misner string. In Sec. IV, we investigate the CA conjecture with the additional Maxwell boundary term. Finally, conclusions are presented in Sec. V.

\section{GEOMETRY OF CHARGED TAUB-NUT-ADS SPACETIME}

In this paper, we consider the charged Taub-NUT-AdS solution of the four-dimensional Einstein-Maxwell gravity, where the bulk action can be written as

$$
I_{\text {bulk }}=\frac{1}{16 \pi} \int_{M} d^{4} x \sqrt{-g}\left(R-2 \Lambda-F_{a b} F^{a b}\right),
$$

in which $\boldsymbol{F}=d \boldsymbol{A}$ is the electromagnetic strength and $R$ is the Ricci scalar of the spacetime. The equations of motion are given by

$$
R_{a b}-\frac{1}{2} R g_{a b}+\Lambda g_{a b}=T_{a b}, \quad d \boldsymbol{G}=0,
$$

with the energy-momentum tensor of the Maxwell field

$$
T_{a b}=F_{a c} F_{b}^{c}+G_{a c} G_{b}^{c},
$$

and

$$
\boldsymbol{G}=\star \boldsymbol{F} .
$$

The electric and magnetic charges inside a two-dimensional surface $S^{2}$ can be defined as

$$
q_{e}\left[S^{2}\right]=\frac{1}{4 \pi} \int_{S^{2}} \boldsymbol{G}, \quad q_{m}\left[S^{2}\right]=\frac{1}{4 \pi} \int_{S^{2}} \boldsymbol{F} .
$$

According to the equations of motion (5), the charged Taub-NUT-AdS solution can be read off $[72,73]$ as

$$
\begin{aligned}
d s^{2}= & -f(r)(d t+2 n \cos \theta d \phi)^{2}+\frac{d r^{2}}{f(r)} \\
& +\left(r^{2}+n^{2}\right)\left(d \theta^{2}+\sin ^{2} \theta d \phi^{2}\right), \\
\boldsymbol{A}= & -\left[h(r)-h_{0}\right] d t-2 h(r) n \cos \theta d \phi,
\end{aligned}
$$

where

$$
f(r)=\frac{r^{2}-2 m r-n^{2}+4 n^{2} g^{2}+e^{2}}{r^{2}+n^{2}}-\frac{3 n^{4}-6 n^{2} r^{2}-r^{4}}{l^{2}\left(r^{2}+n^{2}\right)}
$$

is the blackening factor,

$$
h(r)=\frac{e r}{r^{2}+n^{2}}+\frac{g\left(r^{2}-n^{2}\right)}{r^{2}+n^{2}},
$$

and $h_{0}$ is some arbitrary constant which reflects the gauge freedom of the electromagnetic field. Besides, $n$ and $m$ are the NUT and mass parameters, respectively, $e$ and $g$ are the electric and magnetic parameters, respectively, and $l$ is the anti-de Sitter (AdS) radius with $\Lambda=-3 / l^{2}$. In this paper, we consider only the solution which has two RN-type horizons which are located on the sphere with radius of $r=r_{ \pm}$. And we can see that in this solution, apart from the RN-type horizons, there also exist two Misner string singularities on the north and south pole axes. And this Misner string will affect the topology of this spacetime.

According to Eq. (9), we can immediately obtain the electromagnetic field tensor

$$
\begin{aligned}
\boldsymbol{F}= & -\frac{e\left(n^{2}-r^{2}\right)+4 n^{2} g r}{\left(r^{2}+n^{2}\right)^{2}} d r \wedge d t+\frac{2 n\left[e r+g\left(r^{2}-n^{2}\right)\right]}{r^{2}+n^{2}} \\
& \times \sin \theta d \theta \wedge d \phi-2 n \frac{e\left(n^{2}-r^{2}\right)+4 n^{2} g r}{\left(r^{2}+n^{2}\right)^{2}} \cos \theta d r \wedge d \phi .
\end{aligned}
$$

Then, we can instantly obtain 


$$
\begin{aligned}
\boldsymbol{G}= & \frac{2 n\left[g\left(n^{2}-r^{2}\right)-e r\right]}{\left(r^{2}+n^{2}\right)^{2}} d r \wedge d t+\frac{e\left(r^{2}-n^{2}\right)-4 n^{2} g r}{r^{2}+n^{2}} \\
& \times \sin \theta d \theta \wedge d \phi+\frac{4 n^{2}\left[g\left(n^{2}-r^{2}\right)-e r\right]}{\left(r^{2}+n^{2}\right)^{2}} \cos \theta d r \wedge d \phi .
\end{aligned}
$$

Performing the integration over a sphere $S_{r}$ of radius $r$ according to Eq. (8), we can obtain the electric and magnetic charges inside this sphere:

$$
\begin{aligned}
q_{e}(r) & =\frac{e\left(r^{2}-n^{2}\right)-4 g r n^{2}}{r^{2}+n^{2}}, \\
q_{m}(r) & =\frac{2 n\left[e r+g\left(r^{2}-n^{2}\right)\right]}{r^{2}+n^{2}} .
\end{aligned}
$$

One can note that, disparate with common results of the dyonic solutions, the charges vary with the radius of the sphere $S_{r}$, which can be easily understood, since the sphere $S_{r}$ is not a close surface due to the existence of the Misner singularities. And two kinds of special values of these charges are, respectively, the asymptotic $(r \rightarrow \infty)$ charges:

$$
Q_{e}=e, \quad Q_{m}=2 g n,
$$

and the horizon $\left(r=r_{ \pm}\right)$charges:

$$
Q_{e}^{( \pm)}=q_{e}\left(r_{ \pm}\right), \quad Q_{m}^{( \pm)}=q_{m}\left(r_{ \pm}\right)
$$

where $r_{ \pm}$denote the radius of the outer and inner horizons. We can note that the electromagnetic duality $\left(q_{e}^{2} \leftrightarrow q_{m}^{2}\right)$ can be realized by $e \leftrightarrow-2 n g, 2 n g \leftrightarrow e$. By performing the conformal method [74], the mass of this black hole is obtained by

$$
M=m,
$$

while the total angular momentum of the spacetime vanishes. According to the line element (9), the Killing vector of the horizon is manifestly given by

$$
k^{a}=\left(\frac{\partial}{\partial t}\right)^{a}
$$

And the temperature of these RN-type horizons is given by the surface gravity

$$
\begin{aligned}
T^{( \pm)} & =\frac{\kappa_{ \pm}}{2 \pi}=\frac{f^{\prime}\left(r_{ \pm}\right)}{4 \pi} \\
& =\frac{1}{4 \pi r_{ \pm}}\left(1+\frac{3\left(r_{ \pm}^{2}+n^{2}\right)}{l^{2}}-\frac{e^{2}+4 n^{2} g^{2}}{r_{ \pm}^{2}+n^{2}}\right) .
\end{aligned}
$$

The entropies of these horizons can be identified with the horizon area law:

$$
S^{( \pm)}=\frac{A_{ \pm}}{4}=\pi\left(r_{ \pm}^{2}+n^{2}\right) .
$$

In order to obtain an expected feature of the thermodynamics in this spacetime, Ref. [71] also introduced some quantities related to the Misner string, namely, the Misner potential $\psi$ and Misner charge $N^{( \pm)}$:

$$
\begin{aligned}
\psi= & \psi^{( \pm)}=\frac{1}{8 \pi n} \\
N^{( \pm)}= & -\frac{4 n^{3} \pi}{r_{ \pm}}\left[1+\frac{3\left(n^{2}-r_{ \pm}^{2}\right)}{l^{2}}+\frac{\left(r_{ \pm}^{2}-n^{2}\right)\left(e^{2}+4 e g r_{ \pm}\right)}{\left(r_{ \pm}^{2}+n^{2}\right)^{2}}\right. \\
& \left.-\frac{4 n^{2} g^{2}\left(3 r_{ \pm}^{2}+n^{2}\right)}{\left(r_{ \pm}^{2}+n^{2}\right)^{2}}\right] .
\end{aligned}
$$

Then, the first law of this black hole can be written as

$$
\begin{aligned}
\delta M= & T^{( \pm)} \delta S^{( \pm)}+V^{( \pm)} \delta P \\
& +\phi_{e}^{( \pm)} \delta Q_{e}+\phi_{m}^{( \pm)} \delta Q_{m}^{( \pm)}+\psi \delta N^{( \pm)},
\end{aligned}
$$

where

$$
\begin{gathered}
P=-\frac{\Lambda}{8 \pi}=\frac{3}{8 \pi l^{2}}, \\
V^{( \pm)}=\frac{4}{3} \pi r_{ \pm}^{3}\left(1+\frac{3 n^{2}}{r_{ \pm}^{2}}\right)
\end{gathered}
$$

are the thermodynamic pressure and thermodynamic volume, respectively, of the outer and inner horizons and

$$
\phi_{e}^{( \pm)}=\frac{e r_{ \pm}-2 g n^{2}}{r_{ \pm}^{2}+n^{2}}, \quad \phi_{m}^{( \pm)}=\frac{n\left(2 g r_{ \pm}+e\right)}{r_{ \pm}^{2}+n^{2}}
$$

are the electric and magnetic potentials, respectively, of the RN-type horizons.

\section{COMPLEXITY GROWTH RATE IN ORIGINAL CA CONJECTURE}

In this section, we use the complexity equals action conjecture to evaluate the complexity growth rate of the charged Taub-NUT-AdS black hole of general relativity coupled with a Maxwell field. As suggested by Ref. [13], the CA complexity is equal to the full on-shell action on the WDW patch, which includes not only the bulk action but the surface terms, corner terms, and counterterms as well. According to Ref. [13], the total action is given by

$$
\begin{aligned}
I= & I_{\text {bulk }}+\frac{1}{8 \pi} \int_{\mathcal{B}} d^{3} x \sqrt{|h|} K \pm \frac{1}{8 \pi} \int_{\mathcal{C}} d^{2} x \sqrt{\sigma} \eta \\
& +\frac{1}{8 \pi} \int_{\mathcal{N}} d \lambda d^{2} \theta \sqrt{\gamma} \kappa+\frac{1}{8 \pi} \int_{\mathcal{N}} d \lambda d^{2} \theta \sqrt{\gamma} \Theta \ln \left(\ell_{\mathrm{ct}} \Theta\right),
\end{aligned}
$$



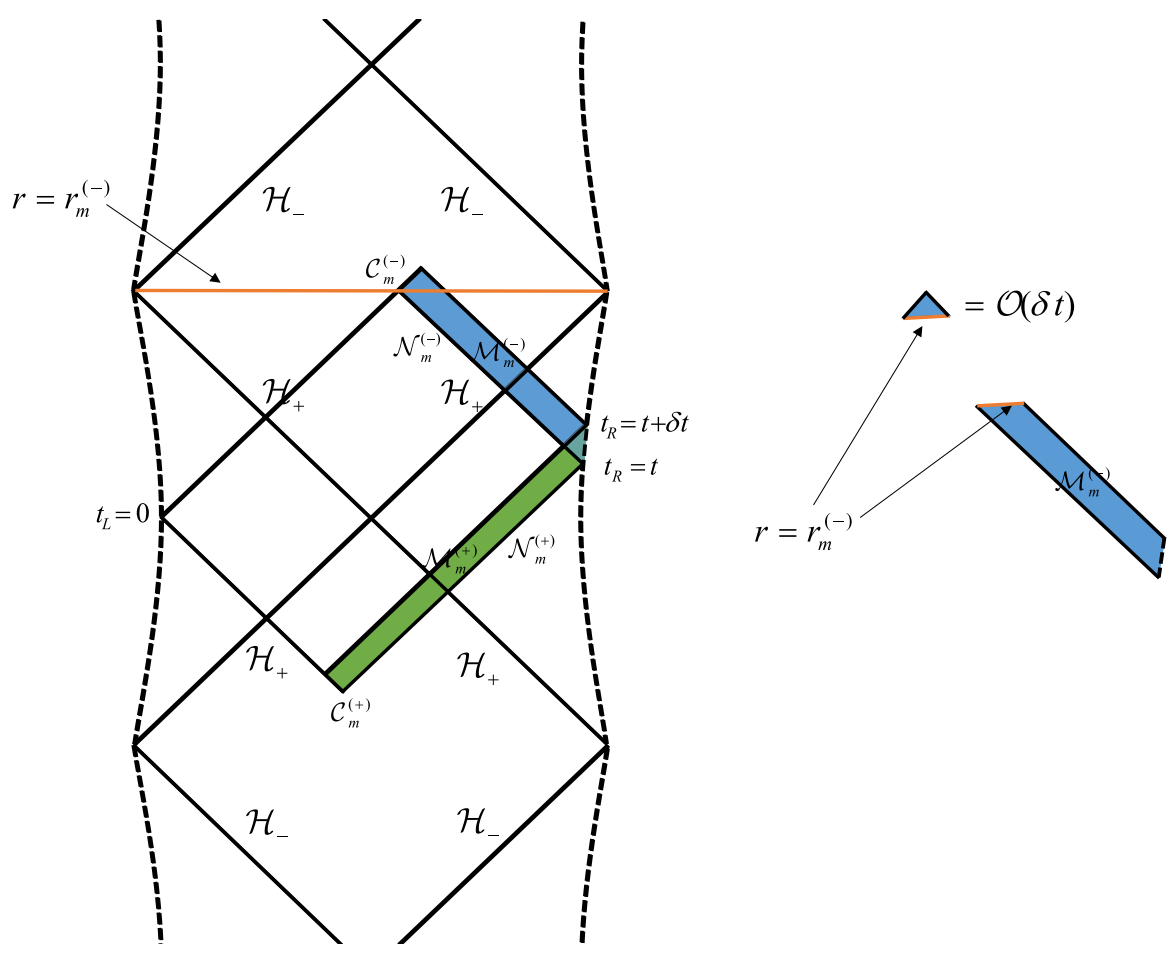

FIG. 1. Wheeler-DeWitt patch of a charged Taub-NUT-AdS black hole with two RN-type horizons, where the dashed lines denote the cutoff surface at asymptotic infinity, satisfying the asymptotic symmetries. In the left panel, we show that, under the first-order approximation of $\delta t$, the bulk region $\delta \mathcal{M}_{m}^{(-)}$can be generated by the Killing vector $k^{a}$ through $\mathcal{N}_{m}^{(-)}$.

where $K=\nabla_{a} n^{a}$ is the trace of the extrinsic curvature, $\lambda$ is the parameter of the null generator $k^{a}$ on the null segment, $\kappa$ measures the failure of $\lambda$ to be an affine parameter which is derived from $k^{a} \nabla_{a} k^{b}=\kappa k^{b}, \Theta=\nabla_{a} k^{a}$ is the expansion scalar, and $\ell_{\mathrm{ct}}$ is an arbitrary length scale. Here the surface term and joint term are introduced to make the variational principle well posed, and the counterterm is added to ensure reparametrization invariance on the null boundaries.

Next, to evaluate the complexity growth rate in the CA context, we consider the change of the action within the WDW patch. By considering the shift symmetry of this spacetime geometry, we can fix the left boundary time $t_{L}=0$ and vary only the right boundary time $t_{R}=t$. Then, the changes of the WDW patch of the charged Taub-NUT-AdS black holes with two RN-type horizons can be illustrated in the Penrose diagram in Fig. 1. To regulate the divergence near the AdS boundary, a cutoff surface $r=r_{\Lambda}$ is introduced. Since we consider only the time growth rate of the complexity, we can neglect the higher-order term of $\delta t$. As illustrated in the right panel in Fig. 1, under the first-order approximation of $\delta t$, the bulk region $\delta \mathcal{M}_{m}^{( \pm)}$can be generated by the Killing vector $k^{a}$ through an asymptotic null hypersurface $\mathcal{N}_{m}^{( \pm)}$which terminates on the 2-sphere $\mathcal{C}_{m}^{( \pm)}$with the radius $r=r_{m}^{( \pm)}$. Without the loss of generality, we shall adopt the affine parameter for the null generator of the null surface; as a consequence, the surface term vanishes on all null boundaries. With these in mind, the change of the total action can be written as

$$
\delta I_{\mathrm{WDW}}=I_{\delta \mathcal{M}_{m}^{(-)}}-I_{\delta \mathcal{M}_{m}^{(+)}}+\delta I_{\mathcal{C}_{m}^{(-)}}-\delta I_{\mathcal{C}_{m}^{(+)}}+\delta I_{\mathrm{ct}}
$$

\section{A. Bulk contributions}

We start by evaluating the contributions from the bulk action $\delta \mathcal{M}_{m}^{(-)}$in the complexity growth rate. For simplification, we will neglect the index for the quantities in this region. For the bulk contribution from the gravitational action, we have

$$
I_{\text {grav }}=\frac{\Lambda}{8 \pi} \int_{\delta \mathcal{M}} \sqrt{-g} d^{4} x=\frac{\Lambda \delta t}{8 \pi} \int_{\mathcal{N}} \star k .
$$

Together with the equations of motion (5), utilizing the facts that $k^{a}$ is a Killing vector and the sum of cyclic permutations of the last three indices in $R^{a}{ }_{b c d}$ vanishes, we have the following identity:

$$
\nabla_{a} \nabla^{a} k^{b}=-R_{a}^{b} k^{a}=-\Lambda k^{b}-T^{a b} k_{a} .
$$

From the equation of motion $d \boldsymbol{G}=0$ of the electromagnetic field, we can see that the on-shell value of $\boldsymbol{G}$ is a closed 2-form, which implies that there exists a 1-form $\boldsymbol{B}$ such that $\boldsymbol{G}=d \boldsymbol{B}$. We can note that the new-defined vector potential $\boldsymbol{B}$ also allows a gauge freedom. Using the solution (9), it is not difficult to verify that this vector potential can be expressed as

$$
\boldsymbol{B}=\left[p(r)-p_{0}\right] d t+2 n p(r) \cos \theta d \phi
$$


with

$$
p(r)=\frac{4 n^{2} g r-e\left(r^{2}-n^{2}\right)}{2 n\left(r^{2}+n^{2}\right)}
$$

and arbitrary constant $p_{0}$ which should be determined when we fix the gauge. Substituting Eq. (6) into (27), the second term becomes

$$
\begin{aligned}
T^{a b} k_{a}= & k_{a} F^{a}{ }_{c} F^{b c}+k_{a} G^{a}{ }_{c} G^{b c} \\
= & F^{b c} k_{a} \nabla^{a} A_{c}-F^{b c} k_{a} \nabla_{c} A^{a}+G^{b c} k_{a} \nabla^{a} B_{c} \\
& -G^{b c} k_{a} \nabla_{c} B^{a} \\
= & -F^{b c} \nabla_{c} \Phi_{e}+G^{b c} \nabla_{c} \Phi_{m} \\
= & \nabla_{a}\left(F^{a b} \Phi_{e}-G^{a b} \Phi_{m}\right),
\end{aligned}
$$

where we have used the fact that Lie derivatives of vector potentials $\boldsymbol{A}$ and $\boldsymbol{B}$ along the vector $k^{a}$ vanish, namely, $\mathcal{L}_{k} \boldsymbol{A}=0$ and $\mathcal{L}_{\boldsymbol{k}} \boldsymbol{B}=0$, and denote the contractions of Killing vector $k^{a}$ with two vector potentials as

$$
\Phi_{e}=k^{a} A_{a}, \quad \Phi_{m}=-k^{a} B_{a},
$$

which can be regarded as the electric and magnetic potentials, respectively, of the Maxwell field. And the counterparts of the inner and outer horizon can be defined, respectively, as

$$
\begin{aligned}
& \phi_{e}^{( \pm)}=\Phi_{e}(\infty)-\Phi_{e}\left(r_{ \pm}\right), \\
& \phi_{m}^{( \pm)}=\Phi_{m}(\infty)-\Phi_{m}\left(r_{ \pm}\right) .
\end{aligned}
$$

From Eqs. (9) and (28), it is not difficult to see that they are actually the potentials as shown in Eq. (23). Then, Eq. (27) together with (30) yields

$$
\nabla_{a}\left(\nabla^{a} k^{b}+F^{a b} \Phi_{e}-G^{a b} \Phi_{m}\right)=-\Lambda k^{b} .
$$

Using the language of differential forms, we have

$$
2 \Lambda \star k=d\left(\star d \boldsymbol{k}+2 \Phi_{e} \boldsymbol{G}+2 \Phi_{m} \boldsymbol{F}\right) .
$$

Substituting the above expression into (26), the gravitational part (26) can be expressed as

$$
I_{\text {grav }}=\frac{\delta t}{16 \pi} \int_{\mathcal{N}} d\left(\star d \boldsymbol{k}+2 \Phi_{e} \boldsymbol{G}+2 \Phi_{m} \boldsymbol{F}\right) .
$$

Next, we consider the bulk contribution from the electromagnetic field,

$$
\begin{aligned}
I_{\mathrm{EM}} & =-\frac{1}{8 \pi} \int_{\delta \mathcal{M}} \boldsymbol{F} \wedge \boldsymbol{G} \\
& =-\frac{\delta t}{8 \pi} \int_{\mathcal{N}} k \cdot(\boldsymbol{F} \wedge \boldsymbol{G}) \\
& =-\frac{\delta t}{8 \pi} \int_{\mathcal{N}}[(k \cdot d \boldsymbol{A}) \wedge \boldsymbol{G}+\boldsymbol{F} \wedge(k \cdot d \boldsymbol{B})] \\
& =\frac{\delta t}{8 \pi} \int_{\mathcal{N}} d\left(\Phi_{e} \boldsymbol{G}-\Phi_{m} \boldsymbol{F}\right),
\end{aligned}
$$

where we also used $\mathcal{L}_{k} \boldsymbol{A}=\mathcal{L}_{k} \boldsymbol{B}=0$, as well as $d \boldsymbol{F}=$ $d \boldsymbol{G}=0$ for the on-shell field $\boldsymbol{A}$. Combing these results, the bulk contribution becomes

$$
\begin{aligned}
I_{\delta \mathcal{M}} & =I_{\text {grav }}+I_{\mathrm{EM}} \\
& =\frac{\delta t}{16 \pi} \int_{\mathcal{N}} d\left(\star d \boldsymbol{k}+4 \Phi_{e} \boldsymbol{G}\right) \\
& =\frac{\delta t}{16 \pi} \int_{\partial \mathcal{N}}\left(\star d \boldsymbol{k}+4 \Phi_{e} \boldsymbol{G}\right) .
\end{aligned}
$$

For the normal black holes, the hypersurface $\mathcal{N}$ is bounded by the spheres $\mathcal{C}$ with the radius $r=r_{m}^{(-)}$and $S_{\infty}$ at asymptotic infinity. However, when the Misner strings are present, there exist the Misner string singularities which locate at $\theta=0$ and $\theta=\pi$. Then, the decomposition will depend on the Misner strings (see Fig. 2). Therefore, we also need to introduce two Misner string tubes $\mathcal{T}_{N}$ and $\mathcal{T}_{S}$ which are located at $\theta=\epsilon$ and $\theta=\pi-\epsilon$ with infinitesimal parameter $\epsilon$, respectively. After taking into account the orientation of the boundaries, we have

$$
\partial \mathcal{N}=\mathcal{T}_{N}+S_{\infty}-\mathcal{T}_{S}-\mathcal{C}
$$

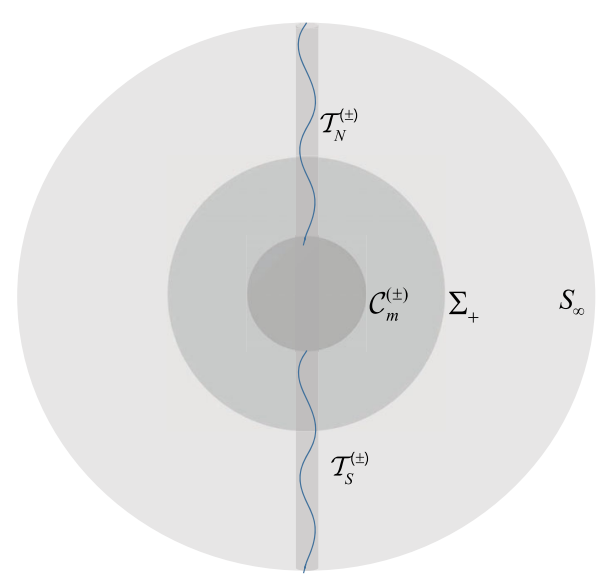

FIG. 2. The null segment $\mathcal{N}_{m}^{( \pm)}$of WDW patch in charged Taub-NUT-AdS spacetimes: Misner tubes, as a result of the effect of NUT parameter $n$, besides the standard boundaries $\mathcal{C}_{m}^{( \pm)}$and at infinity $S_{\infty}$, the two Misner tubes $\mathcal{T}_{N}^{( \pm)}$and $\mathcal{T}_{S}^{( \pm)}$of radius $\epsilon$ surrounding the symmetric strings are located at the north and south pole axes, $\cos \theta= \pm 1$, which connected $\mathcal{C}_{m}^{( \pm)}$and $S_{\infty}$. Here $\Sigma_{ \pm}$denote the portion on the horizon $\mathcal{H}_{ \pm}$. 
Thus, the integral (37) can be decomposed into the following integrations over several pieces of boundaries:

$$
\begin{aligned}
I_{\delta \mathcal{M}}= & \frac{\delta t}{16 \pi} \int_{S_{\infty}} \star d \boldsymbol{k}-\frac{\delta t}{16 \pi} \int_{\mathcal{C}} \star d \boldsymbol{k}+\frac{\delta t}{4 \pi} \int_{S_{\infty}} \Phi_{e} \boldsymbol{G} \\
& -\frac{\delta t}{4 \pi} \int_{\mathcal{C}} \Phi_{e} \boldsymbol{G}+\frac{\delta t}{16 \pi} \int_{\mathcal{T}_{N}}\left(\star d \boldsymbol{k}+4 \Phi_{e} \boldsymbol{G}\right) \\
& -\frac{\delta t}{16 \pi} \int_{\mathcal{T}_{S}}\left(\star d \boldsymbol{k}+4 \Phi_{e} \boldsymbol{G}\right) .
\end{aligned}
$$

By considering line element (9) of this spacetime, we evaluate the Hodge dual of $k^{a}$, namely,

$$
\begin{aligned}
\star d \boldsymbol{k}= & -\frac{2 n f(r)}{r^{2}+n^{2}} d r \wedge(d t+2 n \cos \theta d \phi) \\
& -\sin \theta\left(r^{2}+n^{2}\right) f^{\prime}(r) d \theta \wedge d \phi .
\end{aligned}
$$

Then, we can further obtain

$$
\int_{\mathcal{C}} \star d \boldsymbol{k}=-4 \pi\left(r^{2}+n^{2}\right) f^{\prime}(r)
$$

Considering the fact that $r \rightarrow r_{-}$at the late times, we have

$$
-\frac{1}{16 \pi} \lim _{t \rightarrow \infty} \int_{\mathcal{C}} \star d \boldsymbol{k}=T S
$$

At the same time, we can also obtain

$$
\begin{aligned}
\int_{\mathcal{T}_{N}} \star d \boldsymbol{k}= & -\int_{\mathcal{T}_{S}} \star d \boldsymbol{k} \\
= & -\frac{8 \pi n^{2} r_{\Lambda}}{l^{2}}-\frac{2 \pi^{2}\left(e^{2}+4 g^{2} n^{2}\right)}{n}+\frac{8 \pi n^{2} r}{l^{2}} \\
& +\frac{4 \pi\left[2 m n^{2}+\left(e^{2}+4 g^{2} n^{2}-2 n^{2}\right) r\right]}{r^{2}+n^{2}} \\
& -\frac{32 \pi n^{4} r}{l^{2}\left(r^{2}+n^{2}\right)}+\frac{4 \pi\left(e^{2}+4 g^{2} n^{2}\right) \arctan (r / n)}{n} .
\end{aligned}
$$

Finally, we evaluate the contributions from the electromagnetic field. According to the above calculation, we can see that our final result (39) is independent on the gauge choice of electromagnetic potentials. For later convenience, here we choose the gauge such that $\Phi_{e}\left(r_{-}\right)=0$, i.e.,

$$
\Phi_{e}(r)=\Phi_{e}^{(-)}(r)=\frac{e r_{-}-2 n^{2} g}{n^{2}+r_{-}^{2}}-\frac{e r-2 n^{2} g}{n^{2}+r^{2}} .
$$

Then, we can further obtain

$$
\begin{aligned}
\int_{\mathcal{T}_{N}} 4 \Phi_{e} \boldsymbol{G}= & -\int_{\mathcal{T}_{s}} 4 \Phi_{e} \boldsymbol{G}=\frac{2 \pi^{2}\left(e^{2}+4 g^{2} n^{2}\right)}{n} \\
& -\frac{4 \pi\left(e^{2}+4 g^{2} n^{2}\right) \arctan \left(r_{h} / n\right)}{n} \\
& +\frac{8 n^{2} \pi\left[e^{2} r-4 n^{2} g(e+g r)\right]}{\left(n^{2}+r^{2}\right)^{2}} \\
& -\frac{16 \pi n^{2} \phi_{e}^{(-)}(e+2 g r)}{n^{2}+r^{2}} \\
& -\frac{4 \pi\left[e^{2} r-4 n^{2} g(e-g r)\right]}{n^{2}+r^{2}} .
\end{aligned}
$$

From these results, Eq. (39) can be expressed by

$$
\begin{aligned}
I_{\delta \mathcal{M}} / \delta t= & \frac{\left(r^{2}+n^{2}\right) f^{\prime}(r)}{4}+\phi_{e}^{(-)} Q_{e}-\Phi_{e}^{(-)}(r) q_{e}(r) \\
& +K^{(-)}(r)+\frac{1}{16 \pi} \int_{S_{\infty}} \star d \boldsymbol{k},
\end{aligned}
$$

where we have denoted

$$
\begin{aligned}
K^{( \pm)}(r)= & \frac{1}{8 \pi} \int_{\mathcal{T}_{N}^{( \pm)}}\left(\star d \boldsymbol{k}+4 \Phi_{e}^{( \pm)} \boldsymbol{G}\right) \\
= & \frac{n^{2} r\left(r^{2}-3 n^{2}\right)}{l^{2}\left(n^{2}+r^{2}\right)}+\frac{n^{2} r\left(e^{2}+2 e g r-r^{2}\right)}{\left(n^{2}+r^{2}\right)^{2}}+\frac{m n^{2}}{n^{2}+r^{2}} \\
& -\frac{n^{4}[r+2 g(e+2 g r)]}{\left(n^{2}+r^{2}\right)^{2}}-\frac{2 n^{2} \phi_{e}^{( \pm)}(e+2 g r)}{n^{2}+r^{2}}-\frac{n^{2} r_{\Lambda}}{l^{2}},
\end{aligned}
$$

with

$$
\Phi_{e}^{(+)}(r)=\frac{e r_{+}-2 n^{2} g}{n^{2}+r_{+}^{2}}-\frac{e r-2 n^{2} g}{n^{2}+r^{2}}
$$

such that $\Phi_{e}^{(+)}\left(r_{+}\right)=0$. With a similar calculation, we can also obtain the contributions from $\delta M_{m}^{(+)}$and the growth rate of the bulk action can be read off:

$\frac{d I_{\text {bulk }}}{d t}=\left[\phi_{e}^{(h)} Q_{e}+K^{(h)}-\Phi_{e}^{(h)} q_{e}+\frac{\left(r^{2}+n^{2}\right) f^{\prime}(r)}{4}\right]_{\chi_{+}}^{\chi_{-}}$,

where we denote $\chi_{ \pm} \equiv\left\{h= \pm, r=r_{m}^{( \pm)}\right\}$. By comparing the expressions of the Misner potential and charge (20), together with the fact that $r_{m}^{( \pm)} \rightarrow r_{ \pm}$at late times, we can easily verify

$$
K^{(-)}\left(r_{-}\right)-K^{(+)}\left(r_{+}\right)=\psi\left(N^{(-)}-N^{(+)}\right) .
$$

Together with the fact that $\Phi_{e}^{( \pm)}\left(r_{ \pm}\right)=0$, the late-time growth rate of the bulk action can be expressed as 


$$
\begin{aligned}
\lim _{t \rightarrow \infty} \frac{d I_{\text {bulk }}}{d t}= & T^{(-)} S^{(-)}-T^{(+)} S^{(+)} \\
& +\left(\phi_{e}^{(-)}-\phi_{e}^{(+)}\right) Q_{e}+\psi\left(N^{(-)}-N^{(+)}\right) .
\end{aligned}
$$

\section{B. Joint contributions}

We next calculate the joint contributions from meeting points $\mathcal{C}_{m}^{( \pm)}$. From the line element (9), it is not hard to find that

$$
\begin{aligned}
& k_{1}^{a}=\frac{1}{f(r)}\left(\frac{\partial}{\partial t}\right)^{a}+\left(\frac{\partial}{\partial r}\right)^{a}, \\
& k_{2}^{a}=-\frac{1}{f(r)}\left(\frac{\partial}{\partial t}\right)^{a}+\left(\frac{\partial}{\partial r}\right)^{a}
\end{aligned}
$$

are the affinely null generator of the past right and past left null boundaries separately, which means that the null surface term will vanish under this choice. Then, we can obtain $k_{1} \cdot k_{2}=2 / f$. By using the transformation parameter $\eta=\ln \left(\left|k_{1} \cdot k_{2}\right| / 2\right)$, we have

$$
I_{\mathcal{C}_{m}^{(+)}}=-\frac{1}{2}\left[\left(r_{m}^{(+)}\right)^{2}+n^{2}\right] \ln \left[-f\left(r_{m}^{(+)}\right)\right] .
$$

According to (52), the integral curves of the two generators can be expressed as $t(\lambda)=t+r^{\star}(\lambda), r(\lambda)=\lambda, \theta(\lambda)=\theta$, $\phi(\lambda)=\phi \quad$ and $\quad t(\lambda)=-r^{\star}(\lambda), \quad r(\lambda)=\lambda, \quad \theta(\lambda)=\theta$, $\phi(\lambda)=\phi$, separately. Here we defined the tortoise coordinates

$$
r^{\star}(r)=-\int_{r}^{\infty} \frac{d r}{f(r)},
$$

where this range of integration is chosen to make the coordinate satisfy the boundary condition $\lim _{r \rightarrow \infty} r_{\star}(r)=0$. According to Ref. [11], it can be written as

$$
\begin{aligned}
r^{\star}(r)= & \frac{\ln \left(\left|r-r_{+}\right| / r\right)}{g\left(r_{+}\right)\left(r_{+}-r_{-}\right)}-\frac{\ln \left(\left|r-r_{-}\right| / r\right)}{g\left(r_{-}\right)\left(r_{+}-r_{-}\right)} \\
& -\frac{1}{r_{+}-r_{-}} \int_{r}^{\infty} G(r) d r
\end{aligned}
$$

with

$$
\begin{aligned}
g(r) & =\frac{f(r)}{\left(r-r_{+}\right)\left(r-r_{-}\right)}, \\
G(r) & =\frac{g\left(r_{+}\right) r-g(r) r_{+}}{g\left(r_{+}\right) g(r) r\left(r-r_{+}\right)}-\frac{g\left(r_{-}\right) r-g(r) r_{-}}{g\left(r_{-}\right) g(r) r\left(r-r_{-}\right)} .
\end{aligned}
$$

By using the integral curves of the generator, the radius of the meeting point $\mathcal{C}_{m}^{(+)}$can be obtained by

$$
r^{\star}\left(r_{m}^{(+)}\right)=-\frac{t}{2} .
$$

Then, the change rate of this dynamical point can be read off:

$$
\frac{d r_{m}^{(+)}}{d t}=-\frac{1}{2} f\left(r_{m}^{(+)}\right)
$$

The time derivative of the joint action can be expressed by $\frac{d I_{\mathcal{C}_{m}^{(+)}}}{d t}=\left.\left[\frac{\left(r^{2}+n^{2}\right) f^{\prime}(r)}{4}+\frac{r f(r)}{2} \ln (-f(r))\right]\right|_{r=r_{m}^{(+)}}$.

With a similar consideration, we can further obtain the contribution from the joint $\mathcal{C}_{m}^{(-)}$. And the growth rate of the joint actions can be written as

$$
\frac{d I_{\text {joint }}}{d t}=\left[\frac{\left(r^{2}+n^{2}\right) f^{\prime}(r)}{4}+\frac{r f(r)}{2} \ln (-f(r))\right]_{r_{m}^{(-)}}^{r_{m}^{(+)}},
$$

where we used the relation

$$
r^{\star}\left(r_{m}^{(-)}\right)=\frac{t}{2}
$$

At late times, we have

$$
\lim _{t \rightarrow \infty} \frac{d I_{\text {joint }}}{d t}=T^{(+)} S^{(+)}-T^{(-)} S^{(-)} .
$$

\section{Counterterm contributions}

Finally, we consider the counterterm contributions. We first evaluate the contribution which comes from the past right null boundary with $\lambda$ as the affine parameter, i.e., $k_{1}^{a}=$ $(\partial / \partial \lambda)^{a}$ in (52), which gives rise to the expansion $\Theta=$ $2 r /\left(n^{2}+r^{2}\right)$. Then, the counterterm of the past right null segment can be shown as

$$
I_{\mathrm{ct}}^{(\mathrm{pr})}=\int_{r_{m}^{(+)}}^{r_{\Lambda}} d \lambda r(\lambda) \ln \left(\frac{2 r(\lambda) \ell_{\mathrm{ct}}}{r(\lambda)^{2}+n^{2}}\right) .
$$

By considering that $r(\lambda)=\lambda$, the rate of this counterterm becomes

$$
\frac{d I_{\mathrm{ct}}^{(\mathrm{pr})}}{d t}=\frac{1}{2} r f(r) \ln \left(\frac{2 r l_{\mathrm{ct}}}{r^{2}+n^{2}}\right) .
$$

Again, we can obtain the counterterm contributions from other segments, and the final result is given by

$$
\frac{d I_{\mathrm{ct}}}{d t}=\left[r f(r) \ln \left(\frac{2 r \ell_{\mathrm{ct}}}{r^{2}+n^{2}}\right)\right]_{r_{m}^{(-)}}^{r_{m}^{(+)}} .
$$




\section{Complexity growth}

By summing all the previous results, one can further obtain

$$
\begin{aligned}
\frac{d C_{A}}{d t}= & \frac{1}{\pi \hbar}\left[K^{(h)}+\phi_{e}^{(h)} Q_{e}-\Phi_{e}^{(h)} q_{e}\right. \\
& \left.-\frac{1}{2} r f \ln \left(\frac{4 r^{2} \ell_{\mathrm{ct}}^{2}|f|}{\left(r^{2}+n^{2}\right)^{2}}\right)\right]_{\chi_{+}}^{\chi_{-}} .
\end{aligned}
$$

In Fig. 3, we show the time dependence of the complexity growth rate with the original CA conjecture. This figure shows a similar behavior to the case of the RN-AdS black hole in Ref. [11], in which the late-time value is approached above. Moreover, we can see that, for the case with small NUT and electromagnetic charges, the Lloyd's bound will be violated when we consider the full-time evolution of the complexity. This can be easily understood, since the charged Taub-NUT-AdS black hole will return to the RN-AdS black hole, and the CA complexity in RN-AdS can also violate the Lloyd's bound in some small charge cases [11].

Finally, we consider the late-time result of this holographic complexity. Summing the above results, it can be written as

$\lim _{t \rightarrow \infty} \frac{d C_{A}}{d t}=\frac{1}{\pi \hbar}\left[\left(\phi_{e}^{(-)}-\phi_{e}^{(+)}\right) Q_{e}+\psi\left(N^{(-)}-N^{(+)}\right)\right]$.

We can note that, in this case, the Misner string plays an important role in the holographic complexity. When the Misner string exists, the late-time complexity growth rate is dependent on the Misner potential and Misner charge which is introduced in Ref. [71] to obtain the expected features of the thermodynamics in the charged Taub-NUTAdS black holes. Moreover, differing from the normal charged black holes, where the charges that appeared in the late-time rate of the CA complexity depend only on their values on the horizons, the electric charge here is the total

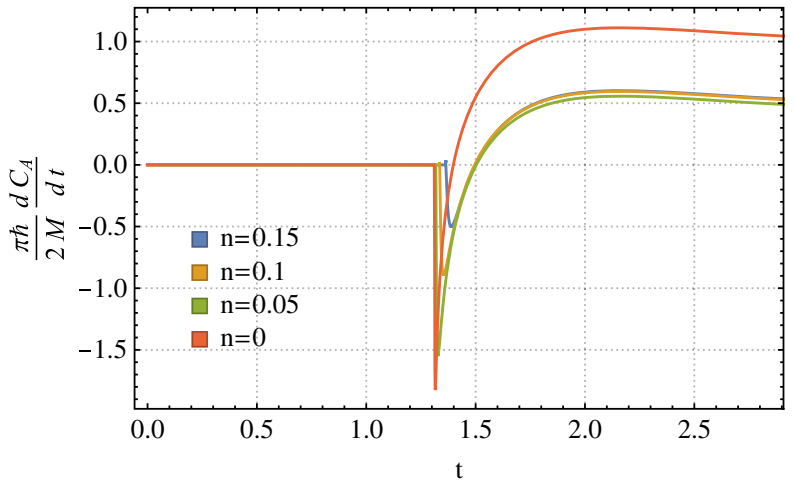

FIG. 3. The time dependence of the complexity growth rate in the context of original CA conjecture with $m=1, l=1$, $\ell_{\mathrm{ct}}=0.1, e=0.2$, and $g=0.2$. charge of the black hole. These results imply that the holographic complexity can exactly reflect the nontrivial topological of this spacetime, such as the existence of the Misner string. Moreover, similar to the dyonic black hole, the late-time growth rate of this original CA conjecture depends only on the electric charge. And one can verify that this result also violates the electromagnetic duality of the Maxwell theory. It was recently proposed in Ref. [51] that this electromagnetic duality can be restored by adding the Maxwell boundary terms. Therefore, in the next section, we would like to study the CA complexity with Maxwell boundary terms.

\section{COMPLEXITY GROWTH RATE WITH MAXWELL BOUNDARY TERM}

In Ref. [51], the authors discussed the CA complexity of the dyonic black hole in Einstein-Maxwell gravity. They found that the original CA complexity in this case also violates the electromagnetic duality, and, for the purely magnetic case, the late-time complexity growth rate always vanishes. In order to obtain the expected features of the CA duality, they suggested that the complexity should be modified by adding the additional Maxwell boundary term

$$
I_{\mu \mathrm{Q}}=\frac{\gamma}{4 \pi} \int_{\partial \mathcal{M}} \boldsymbol{G} \wedge \boldsymbol{A},
$$

where $\gamma$ is a free parameter. Unlike the Gibbons-Hawking surface term, this boundary term was not required by the variation principle, and it gives only different boundary conditions of the electromagnetic field. Then, the full action is given by

$$
I_{\text {full }}=I+I_{\mu \mathrm{Q}} .
$$

If the electromagnetic field satisfies the equation of motion $d \boldsymbol{G}=0$, using the Stokes' theory, this boundary term is equivalent to

$$
I_{\mu \mathrm{Q}}=\int_{\mathcal{M}} \boldsymbol{L}_{\mu \mathrm{Q}}
$$

with

$$
\boldsymbol{L}_{\mu \mathrm{Q}}=\frac{\gamma}{4 \pi} \boldsymbol{G} \wedge \boldsymbol{F} .
$$

With a similar calculation as the EM bulk term (36), the contributions from the Maxwell boundary term to the latetime complexity growth rate can be shown as

$$
\begin{aligned}
\delta I_{\mu \mathrm{Q}}= & -\frac{\gamma \delta t}{4 \pi}\left[\int_{\mathcal{N}_{m}^{(-)}} d\left(\Phi_{e}^{(-)} \boldsymbol{G}-\Phi_{m} \boldsymbol{F}\right)\right. \\
& \left.-\int_{\mathcal{N}_{m}^{(+)}} d\left(\Phi_{e}^{(+)} \boldsymbol{G}-\Phi_{m} \boldsymbol{F}\right)\right] .
\end{aligned}
$$

Without the loss of generality, here we choose the gauge such that $\Phi_{m}(\infty)=0$; i.e., we have $\Phi_{m}\left(r_{ \pm}\right)=-\phi_{m}^{( \pm)}$and 

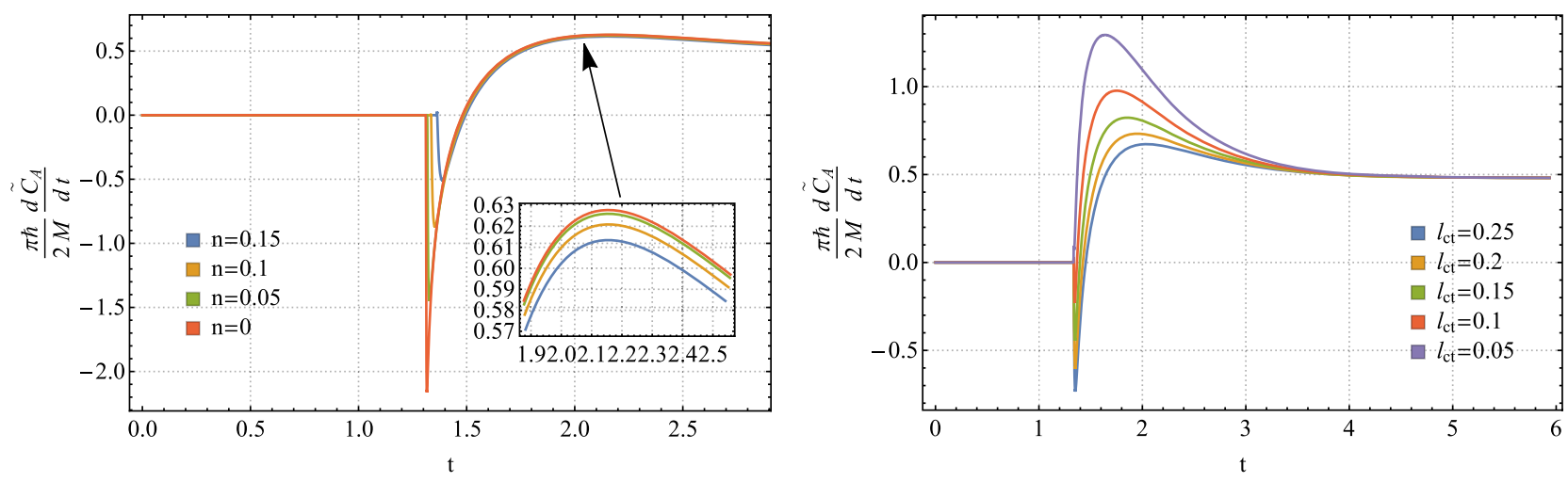

FIG. 4. The time dependence of the complexity growth rate in the context of the CA conjecture with a Maxwell boundary term. In the left panel, we set $m=1, l=1, \ell_{\mathrm{ct}}=0.1, e=0.2$, and $g=0.2$ and change the NUT parameter $n$. In the right panel, we set $m=1$, $l=1, e=0.2, g=0.2$, and $n=0.1$ and change scale length $\ell_{\mathrm{ct}}$.

$$
\Phi_{m}(r)=-\frac{n(e+2 g r)}{n^{2}+r^{2}}
$$

Using Eqs. (11) and (12), we can further obtain

$$
\begin{aligned}
& \Delta_{q}^{( \pm)}\left(r_{m}^{( \pm)}\right) \\
& =\frac{1}{2 \pi} \int_{\mathcal{T}_{N}^{( \pm)}}\left(\Phi_{e}^{( \pm)} \boldsymbol{G}-\Phi_{m} \boldsymbol{F}\right) \\
& =\left.\frac{2 n^{2}\left(r_{h}-r\right)(e+2 g r)\left[e r r_{h}-n^{2}\left(e+2 g r_{h}+2 g r\right)\right]}{\left(n^{2}+r_{h}^{2}\right)\left(n^{2}+r^{2}\right)^{2}}\right|_{\chi_{ \pm}} .
\end{aligned}
$$

Then, we have

$$
\frac{d I_{\mu \mathrm{Q}}}{d t}=\gamma\left[\phi_{e}^{( \pm)} Q_{e}-\Phi_{e}^{( \pm)} q_{e}+\Phi_{m} q_{m}+\Delta_{q}^{(h)}\right]_{\chi_{-}}^{\chi_{+}} .
$$

The complexity growth rate with this boundary term can be expressed by

$$
\begin{aligned}
\frac{d \tilde{C}_{A}}{d t}= & \frac{1}{\pi \hbar}\left[K^{(h)}+(1-\gamma)\left(\phi_{e}^{(h)} Q_{e}-\Phi_{e}^{(h)} q_{e}\right)\right. \\
& \left.+\gamma \Phi_{m} q_{m}+\gamma \Delta_{q}^{(h)}-\frac{1}{2} r f \ln \left(\frac{4 r^{2} \ell_{\mathrm{ct}}^{2}|f|}{\left(r^{2}+n^{2}\right)^{2}}\right)\right]_{\chi_{+}}^{\chi_{-}} .
\end{aligned}
$$

At the late times, it becomes

$$
\begin{aligned}
\lim _{t \rightarrow \infty} \frac{d \tilde{C}_{A}}{d t}= & \frac{1}{\pi \hbar}\left[(1-\gamma)\left(\phi_{e}^{(-)}-\phi_{e}^{(+)}\right) Q_{e}\right. \\
& \left.\gamma\left(\phi_{m}^{(-)} Q_{m}^{(-)}-\phi_{m}^{(+)} Q_{m}^{(+)}\right)+\psi\left(N^{(-)}-N^{(+)}\right)\right] .
\end{aligned}
$$

We can note that, in the charged Taub-NUT-AdS black hole, the Maxwell boundary term affects only the proportion between the electric and magnetic terms, and it does not contribute to the part of the Misner term at late times. Moreover, we can see that, different from the electric charge, here the magnetic charge is determined by the inner and outer horizons. It is not difficult to verify that the case with $\gamma=1 / 2$ also gives the result which satisfies the electromagnetic duality. Then, the complexity growth rate becomes

$$
\begin{aligned}
\frac{d \tilde{C}_{A}}{d t}= & {\left[\frac{2 n^{2} r^{3}-6 n^{4} r+l^{2}\left(2 m n^{2}+e^{2} r-2 n^{2} r+4 n^{2} g^{2} r\right)}{2 \pi \hbar l^{2}\left(n^{2}+r^{2}\right)}\right.} \\
& \left.-\frac{r f(r)}{2 \pi \hbar} \ln \left(-\frac{4 r^{2} \ell_{\mathrm{ct}}^{2} f(r)}{\left(r^{2}+n^{2}\right)^{2}}\right)\right]_{r_{m}^{(+)}}^{r_{m}^{(-)}}
\end{aligned}
$$

Then, its late-time limit can be shown as

$$
\begin{aligned}
\frac{\pi \hbar}{2 M} \lim _{t \rightarrow \infty} \frac{d \tilde{C}_{A}}{d t}= & \frac{\left(r_{+}-r_{-}\right)\left(l^{2}+3 n^{2}+r_{-}^{2}+r_{-} r_{+}+r_{+}^{2}\right)}{2\left(r_{+}+r_{-}\right)\left(l^{2}+6 n^{2}+r_{-}^{2}+r_{+}^{2}\right)} \\
& <\frac{1}{2}
\end{aligned}
$$

which is much less than the Lloyd's bound. In Fig. 4, we show the time dependence of the complexity growth rate in this modified CA conjecture with $\gamma=1 / 2$. This figure also shows a similar behavior to the case of the RN-AdS black hole. However, from the right panel in Fig. 4, we can see that the maximal value of the complexity growth rate will decrease as the length scale $\ell_{\mathrm{ct}}$ decreases and finally approach the late-time value. Therefore, in this case, the Lloyd's bound can always be satisfied as long as we choose a proper length scale $\ell_{\mathrm{ct}}$, since the late-time value is much lower than the Lloyd's bound in this case.

\section{CONCLUSION}

In this paper, we investigated the holographic complexity in the charged Taub-NUT-AdS black holes with two RN-type horizons present in the Einstein-Maxwell gravity. Differing from normal black holes, the Misner string will 
affect the topology of the spacetime geometry. In the context of holography, this nontrivial topology also plays an important role in the holographic complexity. For normal black holes, the late-time rate of the complexity depends only on the quantities on the outer and inner $\mathrm{RN}$-type horizons. However, here it is also determined by the quantities on the Misner string, i.e., the Misner potential $\psi$ and Misner charge $N^{( \pm)}$. Besides, we also found that the electric charge that appeared in the late-time rate is the total charge of this black hole, while, as indicated by the previous literature [6-11], it is only the charges on the horizon for the normal black hole case. These results imply that holographic complexity can exactly reflect the nontrivial topological of this spacetime. Moreover, similar to the dyonic black hole, the late-time growth rate of this original CA conjecture depends only on the electric charge, and it violates the electromagnetic duality of the Maxwell theory. It was recently proposed in Ref. [51] that this electromagnetic duality can be restored by adding the Maxwell boundary terms. Therefore, in Sec. IV, we also studied the CA complexity with the additional Maxwell boundary terms. By adding the Maxwell boundary term, this late-time rate would become sensitive to the magnetic charge. And the special choice $\gamma=1 / 2$ can also make our final result satisfy the electromagnetic duality. Finally, we found that this additional term changes only the proportion between the electric and magnetic charges, and it does not affect the Misner term. Finally, we studied the time dependence of the complexity growth rate with or without a Maxwell boundary term and found that they share similar behaviors with that in RN-AdS black holes. For the special case with $\gamma=1 / 2$, the Lloyd's bound can always be satisfied as long as we choose some proper scale length $\ell_{\mathrm{ct}}$.

\section{ACKNOWLEDGMENTS}

This research was supported by National Natural Science Foundation of China (NSFC) with Grants No. 11375026 and No. 11675015, the Cultivating Program of Excellent Innovation Team of Chengdu University of Technology (Grant No. KYTD201704), the Cultivating Program of Middle-aged Backbone Teachers of Chengdu University of Technology (Grant No. 10912-2019KYGG01511), and the Open Research Fund of Computational Physics Key Laboratory of Sichuan Province, Yibin University (Grant No. JSWL2018KFZ01)
[1] S. Aaronson, The complexity of quantum states and transformations: From quantum money to black holes, arXiv: 1607.05256.

[2] L. Susskind, Computational complexity and black hole horizons, Fortschr. Phys. 64, 24 (2016).

[3] D. Stanford and L. Susskind, Complexity and shock wave geometries, Phys. Rev. D 90, 126007 (2014).

[4] A. R. Brown, D. A. Roberts, L. Susskind, B. Swingle, and Y. Zhao, Holographic Complexity Equals Bulk Action, Phys. Rev. Lett. 116, 191301 (2016).

[5] A. R. Brown, D. A. Roberts, L. Susskind, B. Swingle, and Y. Zhao, Complexity, action, and black holes, Phys. Rev. D 93, 086006 (2016).

[6] J. Jiang, Action growth rate for a higher curvature gravitational theory, Phys. Rev. D 98, 086018 (2018).

[7] R. G. Cai, S. M. Ruan, S. J. Wang, R. Q. Yang, and R. H. Peng, Action growth for AdS black holes, J. High Energy Phys. 09 (2016) 161.

[8] W. D. Guo, S. W. Wei, Y. Y. Li, and Y. X. Liu, Complexity growth rates for AdS black holes in massive gravity and $f(R)$ gravity, Eur. Phys. J. C 77, 904 (2017).

[9] P. Wang, H. Yang, and S. Ying, Action growth in $f(R)$ gravity, Phys. Rev. D 96, 046007 (2017).

[10] W. J. Pan and Y. C. Huang, Holographic complexity and action growth in massive gravities, Phys. Rev. D 95, 126013 (2017).
[11] D. Carmi, S. Chapman, H. Marrochio, R. C. Myers, and S. Sugishita, On the time dependence of holographic complexity, J. High Energy Phys. 11 (2017) 188.

[12] Z. Y. Fan and M. Guo, Holographic complexity and thermodynamics of AdS black holes, Phys. Rev. D 100, 026016 (2019).

[13] L. Lehner, R. C. Myers, E. Poisson, and R. D. Sorkin, Gravitational action with null boundaries, Phys. Rev. D 94, 084046 (2016).

[14] Z. Y. Fan and M. Guo, On the Noether charge and the gravity duals of quantum complexity, J. High Energy Phys. 08 (2018) 031.

[15] Y.S. An, R. G. Cai, and Y. Peng, Time dependence of holographic complexity in Gauss-Bonnet gravity, Phys. Rev. D 98, 106013 (2018).

[16] Y.S. An and R. H. Peng, Effect of the dilaton on holographic complexity growth, Phys. Rev. D 97, 066022 (2018).

[17] A. Reynolds and S. F. Ross, Complexity in de Sitter Space, Classical Quantum Gravity 34, 175013 (2017).

[18] S. Chapman, H. Marrochio, and R. C. Myers, Complexity of formation in holography, J. High Energy Phys. 01 (2017) 062.

[19] X.H. Feng and H.S. Liu, Holographic complexity growth rate in Horndeski theory, Eur. Phys. J. C 79, 40 (2019). 
[20] D. Carmi, R. C. Myers, and P. Rath, Comments on holographic complexity, J. High Energy Phys. 03 (2017) 118.

[21] M. Alishahiha, Holographic complexity, Phys. Rev. D 92, 126009 (2015).

[22] C. A. Agon, M. Headrick, and B. Swingle, Subsystem Complexity and Holography, J. High Energy Phys. 02 (2019) 145.

[23] O. Ben-Ami and D. Carmi, On volumes of subregions in holography and complexity, J. High Energy Phys. 11 (2016) 129.

[24] J. Jiang and M. Zhang, Holographic complexity of the electromagnetic black hole, arXiv:1905.07576.

[25] Y. Zhao, Uncomplexity and black hole geometry, Phys. Rev. D 97, 126007 (2018).

[26] Z. Fu, A. Maloney, D. Marolf, H. Maxfield, and Z. Wang, Holographic complexity is nonlocal, J. High Energy Phys. 02 (2018) 072.

[27] M. Alishahiha, A. Faraji Astaneh, M. R. Mohammadi Mozaffar, and A. Mollabashi, Complexity growth with Lifshitz scaling and hyperscaling violation, J. High Energy Phys. 07 (2018) 042.

[28] J. Couch, S. Eccles, W. Fischler, and M. L. Xiao, Holographic complexity and noncommutative gauge theory, J. High Energy Phys. 03 (2018) 108.

[29] B. Swingle and Y. Wang, Holographic complexity of Einstein-Maxwell-Dilaton gravity, J. High Energy Phys. 09 (2018) 106.

[30] M. Moosa, Evolution of complexity following a global quench, J. High Energy Phys. 03 (2018) 031.

[31] B. Chen, W. M. Li, R. Q. Yang, C. Y. Zhang, and S. J. Zhang, Holographic subregion complexity under a thermal quench, J. High Energy Phys. 07 (2018) 034.

[32] H. S. Liu and H. Lu, Action growth of dyonic black holes and electromagnetic duality, arXiv:1905.06409.

[33] A. Bhattacharyya, A. Shekar, and A. Sinha, Circuit complexity in interacting QFTs and RG flows, J. High Energy Phys. 10 (2018) 140.

[34] T. Ali, A. Bhattacharyya, S. Shajidul Haque, E. H. Kim, and N. Moynihan, Time evolution of complexity: A critique of three methods, J. High Energy Phys. 04 (2019) 087.

[35] T. Ali, A. Bhattacharyya, S. Shajidul Haque, E. H. Kim, and N. Moynihan, Post-quench evolution of distance and uncertainty in a topological system: Complexity, entanglement and revivals, arXiv:1811.05985.

[36] S. A. Hosseini Mansoori, V. Jahnke, M. M. Qaemmaqami, and Y.D. Olivas, Holographic complexity of anisotropic black branes, arXiv:1808.00067.

[37] K. Hashimoto, N. Iizuka, and S. Sugishita, Time evolution of complexity in Abelian gauge theories, Phys. Rev. D 96, 126001 (2017).

[38] R. A. Jefferson and R. C. Myers, Circuit complexity in quantum field theory, J. High Energy Phys. 10 (2017) 107.

[39] S. Chapman, M. P. Heller, H. Marrochio, and F. Pastawski, Towards Complexity for Quantum Field Theory States, Phys. Rev. Lett. 120, 121602 (2018).

[40] R.-Q. Yang, A complexity for quantum field theory states and application in thermofield double states, Phys. Rev. D 97, 066004 (2018).

[41] R. Q. Yang, C. Niu, C. Y. Zhang, and K.-Y. Kim, Comparison of holographic and field theoretic complexities for time dependent thermofield double states, J. High Energy Phys. 02 (2018) 082.

[42] R. Q. Yang, Y. S. An, C. Niu, C. Y. Zhang, and K. Y. Kim, More on complexity of operators in quantum field theory, J. High Energy Phys. 03 (2019) 161.

[43] A. R. Brown and L. Susskind, Second law of quantum complexity, Phys. Rev. D 97, 086015 (2018).

[44] A. P. Reynolds and S. F. Ross, Complexity of the AdS Soliton, Classical Quantum Gravity 35, 095006 (2018).

[45] P. Caputa, N. Kundu, M. Miyaji, T. Takayanagi, and K. Watanabe, Liouville action as path-integral complexity: From continuous tensor networks to AdS/CFT, J. High Energy Phys. 11 (2017) 097.

[46] R. Khan, C. Krishnan, and S. Sharma, Circuit complexity in fermionic field theory, Phys. Rev. D 98, 126001 (2018).

[47] J. Jiang and X. W. Li, Modified complexity equals action conjecture, arXiv:1903.05476.

[48] M. Guo, J. Hernandez, R. C. Myers, and S. M. Ruan, Circuit complexity for coherent states, J. High Energy Phys. 10 (2018) 011.

[49] J. Jiang, J. Shan, and J. Yang, Circuit complexity for free Fermion with a mass quench, arXiv:1810.00537.

[50] R. Q. Yang, Y. S. An, C. Niu, C. Y. Zhang, and K. Y. Kim, Principles and symmetries of complexity in quantum field theory, Eur. Phys. J. C 79, 109 (2019).

[51] K. Goto, H. Marrochio, R. C. Myers, L. Queimada, and B. Yoshida, Holographic complexity equals which action, J. High Energy Phys. 02 (2019) 160.

[52] S. Chapman, J. Eisert, L. Hackl, M. P. Heller, R. Jefferson, H. Marrochio, and R. C. Myers, Complexity and entanglement for thermofield double states, SciPost Phys. 6, 034 (2019).

[53] J. Jiang and X. Liu, Circuit complexity for Fermionic thermofield double states, Phys. Rev. D 99, 026011 (2019).

[54] L. Hackl and R. C. Myers, Circuit complexity for free fermions, J. High Energy Phys. 07 (2018) 139.

[55] R. Nally, Stringy effects and the role of the singularity in holographic complexity, arXiv:1902.09545.

[56] J. Jiang and H. Zhang, Surface term, corner term, and action growth in $\mathrm{F}($ Riemann) gravity theory, Phys. Rev. D 99, 086005 (2019).

[57] J. Jiang and B. X. Ge, Investigating two counting methods of the holographic complexity, Phys. Rev. D 99, 126006 (2019).

[58] S. Chapman, H. Marrochio, and R. C. Myers, Holographic complexity in Vaidya spacetimes. Part I, J. High Energy Phys. 06 (2018) 046.

[59] D. A. Roberts, D. Stanford, and L. Susskind, Localized shocks, J. High Energy Phys. 03 (2015) 051.

[60] S. Chapman, H. Marrochio, and R. C. Myers, Holographic complexity in Vaidya spacetimes. Part II, J. High Energy Phys. 06 (2018) 114.

[61] J. Jiang, Holographic complexity in charged Vaidya black hole, Eur. Phys. J. C 79, 130 (2019).

[62] L. Susskind and Y. Zhao, Switchbacks and the bridge to nowhere, arXiv:1408.2823.

[63] Z. Y. Fan and M. Guo, Holographic complexity under a global quantum quench, arXiv:1811.01473. 
[64] R. G. Cai, M. Sasaki, and S. J. Wang, Action growth of charged black holes with a single horizon, Phys. Rev. D 95, 124002 (2017).

[65] S. S. Hashemi, G. Jafari, A. Naseh, and H. Zolfi, More on complexity in finite cut off geometry, arXiv:1902.03554.

[66] M. Alishahiha, A. Faraji Astaneh, A. Naseh, and M. H. Vahidinia, On complexity for $\mathrm{F}(\mathrm{R})$ and critical gravity, J. High Energy Phys. 05 (2017) 009.

[67] S. Lloyd, Ultimate physical limits to computation, Nature (London) 406, 1047 (2000).

[68] A. H. Taub, Empty space-times admitting a three parameter group of motions, Ann. Math. (N.Y.) 53, 472 (1951).

[69] E. Newman, L. Tamburino, and T. Unti, Empty-space generalization of the Schwarzschild metric, J. Math. Phys. (N.Y.) 4, 915 (1963).
[70] R. A. Hennigar, D. Kubiznak, and R. B. Mann, Thermodynamics of Lorentzian Taub-NUT spacetimes, arXiv:1903 .08668 .

[71] A. B. Bordo, F. Gray, and D. Kubiznak, Thermodynamics and Phase Transitions of NUTty Dyons, J. High Energy Phys. 07 (2019) 119.

[72] C. V. Johnson, The extended thermodynamic phase structure of Taub-NUT and Taub-Bolt, Classical Quantum Gravity 31, 225005 (2014).

[73] N. Alonso-Alberca, P. Meessen, and T. Ortin, Supersymmetry of topological Kerr-Newman-Taub-NUT-AdS spacetimes, Classical Quantum Gravity 17, 2783 (2000).

[74] A. Ashtekar and S. Das, Asymptotically Anti-de Sitter space-times: Conserved quantities, Classical Quantum Gravity 17, L17 (2000). 